\title{
Article
}

\section{Analysis of Decoherence in Linear and Cyclic Quantum Walks}

\author{
Mahesh N. Jayakody ${ }^{1}\left(\mathbb{D}\right.$, Asiri Nanayakkara ${ }^{2}$ iD and Eliahu Cohen ${ }^{1, *(D)}$ \\ 1 The Institute of Nanotechnology and Advanced Materials, Bar Ilan University, Ramat Gan 52900, Israel; \\ jadmn.jayakody@gmail.com \\ 2 National Institute of Fundamental Studies, Kandy 20000, Sri Lanka; asiri.na@nifs.ac.lk \\ * Correspondence: eliahu.cohen@biu.ac.il
}

check for

updates

Citation: Jayakody, M.N.;

Nanayakkara, A.; Cohen, E. Analysis of Decoherence in Linear and Cyclic Quantum Walks. Optics 2021, 2, 236-250. https://doi.org/10.3390/ opt2040022

Academic Editor: Ángel S. Sanz

Received: 11 September 2021

Accepted: 18 October 2021

Published: 22 October 2021

Publisher's Note: MDPI stays neutral with regard to jurisdictional claims in published maps and institutional affiliations.

Copyright: (c) 2021 by the authors. Licensee MDPI, Basel, Switzerland. This article is an open access article distributed under the terms and conditions of the Creative Commons Attribution (CC BY) license (https:/ / creativecommons.org/licenses/by/ $4.0 /)$.

\begin{abstract}
We theoretically analyze the case of noisy Quantum walks (QWs) by introducing four qubit decoherence models into the coin degree of freedom of linear and cyclic QWs. These models include flipping channels (bit flip, phase flip and bit-phase flip), depolarizing channel, phase damping channel and generalized amplitude damping channel. Explicit expressions for the probability distribution of QWs on a line and on a cyclic path are derived under localized and delocalized initial states. We show that QWs which begin from a delocalized state generate mixture probability distributions, which could give rise to useful algorithmic applications related to data encoding schemes. Specifically, we show how the combination of delocalzed initial states and decoherence can be used for computing the binomial transform of a given set of numbers. However, the sensitivity of QWs to noisy environments may negatively affect various other applications based on QWs.
\end{abstract}

Keywords: quantum walks; decoherence; quantum-to-classical transition

\section{Introduction}

Quantum walks (QWs), which differ much from their classical counterparts, have gained substantial attention of the scientific community by becoming an effective ideation and testing ground in various areas of science. Primarily, QWs contribute to theoretical and practical advancement in quantum algorithms [1-4], and quantum computing [5,6] in general. Additionally, they are used to model transport phenomena in biological systems [7-9] and other physical phenomena such as Anderson localization [10-14] and the appearance of topological phases $[15,16]$. Quantum-to-classical transitions in finite dimensional QWs have also been a topic of great interest [8,17-33]. In general, a quantumto-classical transition is triggered by launching interventions on the internal degree of freedom (coin state) $[17,21-27,30,33,34]$ or on the external degree of freedom (position state) $[8,18,29,32,33]$ or on both degrees of freedom $[19,20,29,33]$ of the coin-walker system. Most often, such interventions are introduced in the form of a decoherence model. In this study, we are particularly interested in decoherence models defined on the coin degree of freedom that trigger quantum-to-classical transition in quantum walks on a line and a cycle.

A key feature that distinguishes a classical walk from a QW is the second moment or the variance $\left(\sigma^{2}=\left\langle x^{2}\right\rangle_{t}-\langle x\rangle_{t}^{2}\right)$ of the position probability distribution of the walk. The variance of a classical random walk grows linearly with time $\left(\sigma^{2} \propto t\right)$ [35]. In contrast, the variance of a QW grows quadratically with time $\left(\sigma^{2} \propto t^{2}\right)[28,31]$. Hence, as a rule of thumb, the time dependency of the variance is utilized as a quantitative measure of a quantum-to-classical transition in quantum walks.

In the literature, some possible effects can be recognized that yield classical signature in QWs as a consequence of interventions on the coin degree of freedom. An early practice of such an effort can be found in [17] where a random element is introduced into the coin transformation to switch the walker from quantum-to-classical regime. An obvious way of achieving the classical probabilities in the site occupation of the quantum walker is 
performing a strong projective measurement on the coin state of the walker at each step. The record of measurement outcomes always corresponds to a particular classical path. By averaging over all possible measurement outcomes, one can observe the emergence of classical signature in QWs [23]. Sometimes this is termed the corresponding classical walk of a given QW. Classical signature in QWs can be recovered even without performing a measurement on the coin state at each time step. Instead, one can replace the quantum coin with a new coin operator at every time step. As a result of using different coins at each step, the effect of interference between paths tends to diminish with time. After $n$ steps we have an accumulation of $n$ coins which are entangled with the position of the walker. By performing a measurement on the coin state of the final coin-walker state, it is possible to acquire a unique classical path. The expected classical result can be produced by averaging over all such possible outcomes [22]. In some studies, refs. [20,29] various choices of projections on coin space are used to break the coherence in the system partially and give rise to classical-like behavior. Ref. [27] shows that QWs changeover between quantum and classical regime depending on the strength of the measurement made on the coin state of the walker. The measurement strength is determined via an ancilla that serves as a coin meter. The studies in $[26,30]$ have achieved quantum-to-classical transition by introducing random fluctuations in the coin degree of freedom. A microscopic decoherence model for Hadamard walk is introduced in [24] by attaching a stochastic Hermitian operator to the single-qubit chirality space. Under this decoherence model, drifts and fluctuations occur in the parameters of the total Hamiltonian and as a consequence, Hadamard walk is exposed to a unitary noise and gives classical-like behavior in the long time limit. Phase-space approach in QWs has been used to study the behavior of a quantum walker when the quantum coin interacts with the environment [25]. In [36], the phase-space approach is used to analyze the QW scheme of a single cesium atom moving along a one dimensional optical lattice under the influence of a spin decoherence model. Space and time depended coin operators are used in $[34,37]$ to achieve classical-like behavior in QWs. A phenomenological decoherence model is introduced in [21,23] for QWs on a line by defining a completely positive map on the coin degree of freedom. This model contains decoherence schemes like pure dephasing and weak measurements on coin space and characterizes a QW with a coin subjected to decoherence. Analytical solution for a special case of this model has been derived for the usual Hadamard walk and hence it has been proved that quantumto-classical transition of the Hadamard walk occurs only in the asymptotic limit. In [33], the aforementioned phenomenological decoherence model is generalized to all kinds of decoherence that include coin, position and coin-position decoherence.

Decoherence is considered as a factor that diminishes the resourcefulness of quantum systems. This is true for photons as well as for matter waves, whether they are utilized for quantum walks or other applications. However, decoherence in QWs has shown some possible useful applications [20,36]. Diversified photonic realizations of quantum walks have appeared in the literature with various kinds of tunable decoherence [12,38-40], while some realizations, like the one in [38], could display negligible decoherence, others like [40] did have to face the implications of decoherence due to dephasing induced by setup misalignment. Motivated by both challenges and opportunities, we intended to explore QWs subjected to decoherence. Specifically, we wished to seek any possible application related to quantum simulations or quantum information processing. We consider four special cases of the general decoherence model given in [21,23]. These models include flipping channels (bit flip, phase flip and bit-phase flip), depolarizing channel, phase damping and amplitude damping. The influence of these decoherence models on linear and cyclic QWs is studied separately, but general conclusions are eventually drawn.

\section{Quantum Walk on a Line}

Let us consider the standard model of QW on line which comprises a two-state coin and a walker. The evolution of the coin-walker system is governed by a unitary operator $U$ defined on the tensor product of two Hilbert spaces, $H_{x} \otimes H_{c}$ which are spanned by 
the position basis $\{|x\rangle\}_{x \in \mathbb{Z}}$ and the coin basis $\{|c\rangle\}_{c \in\{0,1\}}$, respectively. The single-step progression of the system is a sequential process in which the coin is tossed at first and then the walker is moved either to right $(R)$ or left $(L)$ conditional upon the outcome of the coin. Mathematically, the evolution of the system can be represented by a combination of a shift operator $(S)$ that acts on the position of the particle, two projectors $P_{R}=|0\rangle\langle 0|$ and $P_{L}=|1\rangle\langle 1|$ on coin Hilbert space that forms a complete orthogonal system of complimentary projections and a coin operator $(C)$. Hence, a single-step evolution operator of the QW is given by

$$
U=S\left(\mathbb{I}_{x} \otimes C\right),
$$

where $C$ and $S$ are the coin and shift operators, respectively, and $\mathbb{I}_{x}$ is the identity operator in position space. In this study, we consider the following form of coin operator

$$
C=\left(\begin{array}{cc}
\cos \theta & \sin \theta \\
\sin \theta & -\cos \theta
\end{array}\right)
$$

where $\theta \in[0,2 \pi)$. The shift operator is defined as

$$
S=\sum_{x=-\infty}^{\infty}|x+1\rangle\langle x|\otimes| 0\rangle\langle 0|+| x-1\rangle\langle x|\otimes| 1\rangle\langle 1| .
$$

For the sake of convenience we conduct our analysis in momentum space. The transformation from the $x$-basis to $k$-basis is given by

$$
|k\rangle=\sum_{x} e^{i k x}|x\rangle,
$$

where $k \in[-\pi, \pi]$. The inverse transformation is given by

$$
|x\rangle=\frac{1}{2 \pi} \int_{-\pi}^{\pi} d k e^{-i k x}|k\rangle .
$$

Thus in momentum space, $U$ is represented by the unitary operator $U_{k}$ given by

$$
U_{k}=\left(e^{-i k} P_{R}+e^{i k} P_{L}\right) C
$$

This representation allows us to view a single-step evolution of an arbitrary state of the coin-walker system as a transformation performed only on the coin state of that arbitrary state.

\section{Modeling Decohrence on One Dimensional QWs}

\subsection{Localized Initial State}

Consider a one-dimensional QW scheme in which a completely positive (CP) map is performed on the coin degree of freedom at each step before performing the unitary flip of the coin. A CP map is defined in such a way that it introduces a probability mixture of unitary transformations on the coin degree of freedom. Such a scheme resembles a QW with a single coin subjected to decoherence. Probability mixture in CP map directly gives rise to a mixed quantum state and hence urges the need of employing the density operators of quantum sates in the analysis. Let $\left\{\hat{A}_{n}\right\}_{n}$ be a set of operators corresponding to the coin degree of freedom that satisfy the condition $\sum_{n} \hat{A}_{n}^{+} \hat{A}_{n}=\mathbb{I}$. Suppose the walker commences the walk from origin with the state $|0\rangle \otimes\left|\phi_{0}\right\rangle$ where $|0\rangle$ is the initial position state and $\left|\phi_{0}\right\rangle=\alpha|0\rangle+\beta|1\rangle$ is the initial coin state where $\alpha, \beta \in \mathbb{C}$ such that $|\alpha|^{2}+|\beta|^{2}=1$. Then the density operator of the initial state in momentum representation can be written as

$$
\rho_{0}=\int_{-\pi}^{\pi} \frac{d k}{2 \pi} \int_{-\pi}^{\pi} \frac{d k^{\prime}}{2 \pi}|k\rangle\left\langle k^{\prime}|\otimes| \phi_{0}\right\rangle\left\langle\phi_{0}\right|,
$$

where $\left|\phi_{0}\right\rangle\left\langle\phi_{0}\right|$ is the density operator for the initial coin state. After a single step, the state becomes 


$$
\rho_{1}=\int_{-\pi}^{\pi} \frac{d k}{2 \pi} \int_{-\pi}^{\pi} \frac{d k^{\prime}}{2 \pi}|k\rangle\left\langle k^{\prime}\left|\otimes \sum_{n} U_{k} \hat{A}_{n}\right| \phi_{0}\right\rangle\left\langle\phi_{0}\right| \hat{A}_{n}^{\dagger} U_{k^{\prime}}{ }^{\dagger},
$$

where $U_{k}$ is the unitary operator of QW on a line in momentum space and $\hat{A}_{n}$ are Kraus operators. The state at time $t$ can be written as,

$$
\begin{gathered}
\rho_{t}=\int_{-\pi}^{\pi} \frac{d k}{2 \pi} \int_{-\pi}^{\pi} \frac{d k^{\prime}}{2 \pi}|k\rangle\left\langle k^{\prime}\right| \\
\otimes \sum_{n_{1} \ldots n_{t}} U_{k} \hat{A}_{n_{t}} \ldots U_{k} \hat{A}_{n_{1}}\left|\phi_{0}\right\rangle\left\langle\phi_{0}\right| \hat{A}_{n_{1}}^{\dagger} U_{k^{\prime}}{ }^{\dagger} \ldots \hat{A}_{n_{t}}^{\dagger} U_{k^{\prime}}{ }^{\dagger} .
\end{gathered}
$$

In terms of a superoperator $\mathcal{L}_{\hat{A}, k, k^{\prime}}$ defined on the coin degree of freedom we can write the state of the coin-walker system at time $t$ in a compact form as follows:

$$
\rho_{t}=\int_{-\pi}^{\pi} \frac{d k}{2 \pi} \int_{-\pi}^{\pi} \frac{d k^{\prime}}{2 \pi}|k\rangle\left\langle k^{\prime}\left|\otimes \mathcal{L}_{\hat{A}, k, k^{\prime}}^{t}\right| \phi_{0}\right\rangle\left\langle\phi_{0}\right|,
$$

where $\mathcal{L}_{\hat{A}, k, k^{\prime}}\left|\phi_{0}\right\rangle\left\langle\phi_{0}\left|=\sum_{n} U_{k} \hat{A}_{n}\right| \phi_{0}\right\rangle\left\langle\phi_{0}\right| \hat{A}_{n}^{\dagger} U_{k^{\prime}}{ }^{\dagger}$.

\subsection{Delocalized Initial State}

Suppose that a one dimensional $\mathrm{QW}$ is allowed to evolve stating from an arbitrary delocalized initial state of the form $\left|\psi_{0}\right\rangle=\sum_{x}\left(|x\rangle \otimes\left(a_{x}|0\rangle+b_{x}|1\rangle\right)\right)$ where $\sum_{x}\left|a_{x}\right|^{2}+\left|b_{x}\right|^{2}=1$. The density matrix that represents the initial coin-walker system is given by

$$
\begin{aligned}
\rho_{0} & =\left|\psi_{0}\right\rangle\left\langle\psi_{0}\right| \\
& =\sum_{x, x^{\prime}}|x\rangle\left\langle x^{\prime}\right| \otimes\left(a_{x} a_{x^{\prime}}^{*}|0\rangle\left\langle 0\left|+a_{x} b_{x^{\prime}}^{*}\right| 0\right\rangle\left\langle 1\left|+b_{x} a_{x^{\prime}}^{*}\right| 1\right\rangle\left\langle 0\left|+b_{x} b_{x^{\prime}}^{*}\right| 1\right\rangle\langle 1|\right) .
\end{aligned}
$$

In momentum representation $\rho_{0}$ takes the following from

$$
\begin{aligned}
\rho_{0}= & \sum_{x, x^{\prime}} \int_{-\pi}^{\pi} \frac{d k}{2 \pi} \int_{-\pi}^{\pi} \frac{d k^{\prime}}{2 \pi} e^{-i k x} e^{i k^{\prime} x^{\prime}}|k\rangle\left\langle k^{\prime}\right| \\
& \otimes\left(a_{x} a_{x^{\prime}}^{*}|0\rangle\left\langle 0\left|+a_{x} b_{x^{\prime}}^{*}\right| 0\right\rangle\left\langle 1\left|+b_{x} a_{x^{\prime}}^{*}\right| 1\right\rangle\left\langle 0\left|+b_{x} b_{x^{\prime}}^{*}\right| 1\right\rangle\langle 1|\right) .
\end{aligned}
$$

Following (10) the state after time $t$ can be written as

$$
\begin{aligned}
\rho_{t}= & \sum_{x, x^{\prime}} \int_{-\pi}^{\pi} \frac{d k}{2 \pi} \int_{-\pi}^{\pi} \frac{d k^{\prime}}{2 \pi} e^{-i k x} e^{i k^{\prime} x^{\prime}}|k\rangle\left\langle k^{\prime}\right| \\
& \otimes \mathcal{L}_{\hat{A}, k, k^{\prime}}^{t}\left(a_{x} a_{x^{\prime}}^{*}|0\rangle\left\langle 0\left|+a_{x} b_{x^{\prime}}^{*}\right| 0\right\rangle\left\langle 1\left|+b_{x} a_{x^{\prime}}^{*}\right| 1\right\rangle\left\langle 0\left|+b_{x} b_{x^{\prime}}^{*}\right| 1\right\rangle\langle 1|\right) .
\end{aligned}
$$

\section{Decoherence Models}

In this study, we consider four types of qubit decoherence models named, flipping channels (bit flip, phase flip and bit-phase flip), depolarizing channel, phase damping and generalized amplitude damping [41]. The bit flip channel randomly alters the coin basis from one state to the other and the phase flip channel modifies the phase relation between the coin basis states by introducing a random phase factor. The simultaneous effects of a bit and phase flip channels are given by the bit-phase flip channel. In addition, the depolarizing channel allows all the flipping channels to exist with equal probability. The Kraus operators that correspond to flipping and depolarizing channels can be written as follows 


$$
\begin{aligned}
& \mathbf{A}_{0}=\sqrt{p}\left(\begin{array}{ll}
1 & 0 \\
0 & 1
\end{array}\right), \\
& \mathbf{A}_{1}=\sqrt{q}\left(\begin{array}{ll}
0 & 1 \\
1 & 0
\end{array}\right), \\
& \mathbf{A}_{2}=\sqrt{r}\left(\begin{array}{cc}
1 & 0 \\
0 & -1
\end{array}\right), \\
& \mathbf{A}_{3}=\sqrt{s}\left(\begin{array}{cc}
0 & -i \\
i & 0
\end{array}\right),
\end{aligned}
$$

where $p, q, r, s \in \mathbb{R}_{>0}$ such that $p+q+r+s=1$. When $r=s=0$, the model is termed bit flip channel. For the condition of $q=s=0$, we name the model phase flip channel. When $q=r=0$, the model is identified as a bit-phase flip channel. For the criterion of $q=r=s$, the decoherence model in (14) is called as the depolarizing channel [41].

The description of energy dissipation from a qubit is depicted by the phase and generalized amplitude damping models. A noise process that results in the loss of quantum information (relative phase between states) without loss of energy is defined by the phase damping model. By using the unitary freedom in the operator-sum representation, one can prove that the Kraus operators corresponding to phase damping is equivalent to that of phase flip channel [41]. The generalized amplitude damping channel describes the energy relaxation processes of a qubit from an excited state to the ground state due to its surrounding. Kraus operators that corresponds to generalized amplitude damping is defined as follows

$$
\begin{aligned}
& \mathbf{E}_{0}=\sqrt{p}\left(\begin{array}{cc}
1 & 0 \\
0 & \sqrt{1-\gamma}
\end{array}\right), \\
& \mathbf{E}_{1}=\sqrt{p}\left(\begin{array}{cc}
0 & \sqrt{\gamma} \\
0 & 0
\end{array}\right), \\
& \mathbf{E}_{2}=\sqrt{1-p}\left(\begin{array}{cc}
\sqrt{1-\gamma} & 0 \\
0 & 1
\end{array}\right), \\
& \mathbf{E}_{3}=\sqrt{1-p}\left(\begin{array}{cc}
0 & 0 \\
\sqrt{\gamma} & 0
\end{array}\right),
\end{aligned}
$$

where $p \in[0,1]$ is a parameter which could be related to the temperature of the environment and $\gamma \in[0,1]$ could be related to the probability of losing/absorbing a photon. For the condition of $p=0$ or $p=1$, the channel is termed as the amplitude damping channel $[41,42]$. Now let us explore evolution of the quantum walk on a line under the influence of these decoherence channels. Let $O$ be the density operator for the initial coin state. A convenient representation of $O$ is given by

$$
O=r_{0} \sigma_{0}+r_{1} \sigma_{1}+r_{2} \sigma_{2}+r_{3} \sigma_{3}
$$

where $r_{i} \in \mathbb{C}, \sigma_{0}=\mathbb{I}$ and $\sigma_{1,2,3}=\sigma_{x, y, z}$ are the usual Pauli matrices. This representation allows us to express $O$ as a column vector of the form

$$
O=\left(\begin{array}{llll}
r_{0} & r_{1} & r_{2} & r_{3}
\end{array}\right)^{T} .
$$

From (10) the action of the superoperator $\mathcal{L}_{\hat{A}, k, k^{\prime}}$ on $O$ can be written in the Pauli basis as

$$
\mathcal{L}_{\hat{A}, k, k^{\prime}} O=\left(\begin{array}{cccc}
a_{11} & a_{12} & 0 & a_{14} \\
0 & a_{22} & a_{23} & a_{24} \\
0 & a_{32} & a_{33} & a_{34} \\
a_{41} & a_{42} & 0 & a_{44}
\end{array}\right)\left(\begin{array}{c}
r_{0} \\
r_{1} \\
r_{2} \\
r_{3}
\end{array}\right),
$$




$$
\begin{aligned}
& \text { where } a_{11}=(p+q+r+s) \cos \left(k-k^{\prime}\right) \\
& a_{12}=-i(p+q-r-s) \sin \left(k-k^{\prime}\right) \sin 2 \theta \\
& a_{14}=-i(p-q+r-s) \cos 2 \theta \sin \left(k-k^{\prime}\right) \\
& a_{22}=-(p+q-r-s) \cos \left(k+k^{\prime}\right) \cos 2 \theta \\
& a_{23}=(p-q-r+s) \sin \left(k+k^{\prime}\right) \\
& a_{24}=(p-q+r-s) \cos \left(k+k^{\prime}\right) \sin 2 \theta \\
& a_{32}=-(p+q-r-s) \cos 2 \theta \sin \left(k+k^{\prime}\right) \\
& a_{33}=-(p-q-r+s) \cos \left(k+k^{\prime}\right) \\
& a_{34}=(p-q+r-s) \sin \left(k+k^{\prime}\right) \sin 2 \theta \\
& a_{41}=-i(p+q+r+s) \sin \left(k-k^{\prime}\right) \\
& a_{42}=(p+q-r-s) \cos \left(k-k^{\prime}\right) \sin 2 \theta \\
& a_{44}=(p-q+r-s) \cos \left(k-k^{\prime}\right) \cos 2 \theta .
\end{aligned}
$$

Similarly, the superoperator corresponding to generalized amplitude damping channel can be expressed in the Pauli basis as follows

$$
\mathcal{L}_{\hat{E}, k, k^{\prime}} O=\left(\begin{array}{cccc}
e_{11} & e_{12} & 0 & e_{14} \\
e_{21} & e_{22} & e_{23} & e_{24} \\
e_{31} & e_{32} & e_{33} & e_{34} \\
e_{41} & e_{42} & 0 & e_{44}
\end{array}\right) \cdot\left(\begin{array}{c}
r_{0} \\
r_{1} \\
r_{2} \\
r_{3}
\end{array}\right),
$$

where $e_{11}=\cos \left(k-k^{\prime}\right)+i \gamma(1-2 p) \cos 2 \theta \sin \left(k-k^{\prime}\right)$

$e_{12}=-i \sqrt{1-\gamma} \sin 2 \theta \sin \left(k-k^{\prime}\right)$

$e_{14}=i(\gamma-1) \cos 2 \theta \sin \left(k-k^{\prime}\right)$

$e_{21}=\gamma(2 p-1) \sin 2 \theta \cos \left(k+k^{\prime}\right)$

$e_{22}=-\sqrt{1-\gamma} \cos 2 \theta \cos \left(k+k^{\prime}\right)$

$e_{23}=\sqrt{1-\gamma} \sin \left(k+k^{\prime}\right)$

$e_{24}=-(\gamma-1) \sin 2 \theta \cos \left(k+k^{\prime}\right)$

$e_{31}=\gamma(2 p-1) \sin 2 \theta \sin \left(k+k^{\prime}\right)$

$e_{32}=-\sqrt{1-\gamma} \cos 2 \theta \sin \left(k+k^{\prime}\right)$

$e_{33}=-\sqrt{1-\gamma} \cos \left(k+k^{\prime}\right)$

$e_{34}=-(\gamma-1) \sin 2 \theta \sin \left(k+k^{\prime}\right)$

$e_{41}=\gamma(2 p-1) \cos 2 \theta \cos \left(k-k^{\prime}\right)-i \sin \left(k-k^{\prime}\right)$

$e_{42}=\sqrt{1-\gamma} \sin 2 \theta \cos \left(k-k^{\prime}\right)$

$e_{44}=-(\gamma-1) \cos 2 \theta \cos \left(k-k^{\prime}\right)$.

The time evolution of the QW under the influence of decoherence can be achieved by taking the $t$ th power of $\mathcal{L}_{\hat{A}, k, k^{\prime}}$ and $\mathcal{L}_{\hat{E}, k, k^{\prime}}$ superoperators given in (18) and (19). As the complexity of the elements in $\mathcal{L}_{\hat{A}, k, k^{\prime}}$ and $\mathcal{L}_{\hat{E}, k, k^{\prime}}$ matrices grow with the power, it is inconvenient to write an analytical form for each element as a function of time $t$. However, for special cases, one can write such explicit forms for matrix elements in time $t$.

\subsection{Model 1}

Let us consider a scenario in which the influence of no-flip, bit flip, phase flip and bitphase flip decoherence models is equally probable [43]. Then we have $p=q=r=s=\frac{1}{4}$. Hence, the $t$ th power of the operator $\mathcal{L}_{\hat{A}, k, k^{\prime}} \mathrm{O}$ is given by

$$
\mathcal{L}_{\hat{A}, k, k^{\prime}}^{t} O=\left(\begin{array}{cccc}
a_{11}(t) & 0 & 0 & 0 \\
0 & 0 & 0 & 0 \\
0 & 0 & 0 & 0 \\
a_{41}(t) & 0 & 0 & 0
\end{array}\right)\left(\begin{array}{c}
r_{0} \\
r_{1} \\
r_{2} \\
r_{3}
\end{array}\right),
$$

where $a_{11}(t)=\cos ^{t}\left(k-k^{\prime}\right)$ and $a_{41}(t)=-i \cos ^{t-1}\left(k-k^{\prime}\right) \sin \left(k-k^{\prime}\right)$. By rewriting $\mathcal{L}_{\hat{A}, k, k^{\prime}}^{t} O$ in the Pauli basis we obtain

$$
\mathcal{L}_{\hat{A}, k, k^{\prime}}^{t} O=r_{0}\left(\begin{array}{cc}
a_{11}(t)+a_{41}(t) & 0 \\
0 & a_{11}(t)-a_{41}(t)
\end{array}\right) .
$$


Note that the trace of the matrix given in (21) solely depends on the element $a_{11}(t)$ of the superoperator $\mathcal{L}_{\hat{A}, k, k^{\prime}}$ and the coefficient $r_{0}$ of the initial coin state.

\subsection{Model 2}

Now let us consider a scenario in which the probability $\gamma$ of losing/absorbing a photon by the coin qubit of the QW is unity. Then we have $\gamma=1$. Hence, the $t$ th power of the operator $\mathcal{L}_{\hat{E}, k, k^{\prime}} \mathrm{O}$ is given by

$$
\mathcal{L}_{\hat{E}, k, k^{\prime}}^{t} O=\left(\begin{array}{cccc}
e_{11}(t) & 0 & 0 & 0 \\
e_{21}(t) & 0 & 0 & 0 \\
e_{31}(t) & 0 & 0 & 0 \\
e_{41}(t) & 0 & 0 & 0
\end{array}\right) \cdot\left(\begin{array}{c}
r_{0} \\
r_{1} \\
r_{2} \\
r_{3}
\end{array}\right)
$$

where $e_{11}(t)=\left(\Omega_{k, k^{\prime}}\right)^{t}$

$$
\begin{aligned}
& e_{21}(t)=(2 p-1) \sin 2 \theta \cos \left(k+k^{\prime}\right)\left(\Omega_{k, k^{\prime}}\right)^{t-1} \\
& e_{31}(t)=(2 p-1) \sin 2 \theta \sin \left(k+k^{\prime}\right)\left(\Omega_{k, k^{\prime}}\right)^{t-1} \\
& e_{41}(t)=\left((2 p-1) \cos 2 \theta \cos \left(k-k^{\prime}\right)-i \sin \left(k-k^{\prime}\right)\right)\left(\Omega_{k, k^{\prime}}\right)^{t-1} \\
& \Omega_{k, k^{\prime}}=\left(\cos \left(k-k^{\prime}\right)+i(1-2 p) \cos 2 \theta \sin \left(k-k^{\prime}\right)\right) .
\end{aligned}
$$

By rewriting $\mathcal{L}_{\hat{E}, k, k^{\prime}}^{t} O$ in the Pauli basis we obtain

$$
\mathcal{L}_{\hat{E}, k, k^{\prime}}^{t} O=r_{0}\left(\begin{array}{cc}
e_{11}(t)+e_{41}(t) & e_{21}(t)-i e_{31}(t) \\
e_{21}(t)+i e_{31}(t) & e_{11}(t)-e_{41}(t)
\end{array}\right)
$$

Note that the trace of the matrix given in (23) solely depends on the element $e_{11}(t)$ of the superoperator $\mathcal{L}_{\hat{E}, k, k^{\prime}}$ and the coefficient $r_{0}$ of the initial coin state.

\section{Position Probability Distribution}

In this section, we present the position probability distribution of QWs on a line under decoherence. We derive exact formulas for the probability distribution subjected to the decoherence given in Model 1 and Model 2. Under each decoherence model, separate analysis has been done for localized and delocalized initial coin states.

\subsection{Model 1 with Localized Initial State (M1L)}

Let us trace out the coin space from the density matrix of the coin-walker system given in (10). Then we can write the position density matrix under decoherence Model 1 with localized initial state $\rho_{M 1 L}^{(x)}(t)$ at time $t$ as follows

$$
\rho_{M 1 L}^{(x)}(t)=\operatorname{Tr}_{c}\left(\rho_{t}\right)=\int_{-\pi}^{\pi} \frac{d k}{2 \pi} \int_{-\pi}^{\pi} \frac{d k^{\prime}}{2 \pi}|k\rangle\left\langle k^{\prime}\right| \operatorname{Tr}_{c}\left(\mathcal{L}_{\hat{A}, k, k^{\prime}}^{t}\left|\phi_{0}\right\rangle\left\langle\phi_{0}\right|\right) .
$$

The term $\operatorname{Tr}_{\mathcal{C}}\left(\mathcal{L}_{\hat{A}, k, k^{\prime}}^{t}\left|\phi_{0}\right\rangle\left\langle\phi_{0}\right|\right)$ can be evaluated by summing the diagonal terms of the matrix given in (21). Hence, by rewriting the position density matrix $\rho_{M 1 L}^{(x)}(t)$ in $x$-basis, we obtain the following expression

$$
\rho_{M 1 L}^{(x)}(t)=\frac{2 r_{0}}{(2 \pi)^{2}} \sum_{x, x^{\prime}} \int_{-\pi}^{\pi} d k \int_{-\pi}^{\pi} d k^{\prime} e^{i k x} e^{-i k^{\prime} x^{\prime}} \cos ^{t}\left(k-k^{\prime}\right)|x\rangle\left\langle x^{\prime}\right| .
$$

By decomposing the coin state of the localized initial state in the Pauli basis, one can easily show that $r_{0}=\frac{1}{2}$. The definite integrals given in (25) can be evaluated by expressing the cosine function in terms of complex exponential functions and then by applying the binomial theorem. In doing so we obtain the following expression for $\rho_{M 1 L}^{(x)}(t)$ as 


$$
\rho_{M 1 L}^{(x)}(t)=\frac{1}{2^{t}} \sum_{x, x^{\prime}} \sum_{l=0}^{t}\left(\begin{array}{l}
t \\
l
\end{array}\right)\left(\frac{\sin [\pi(x+t-2 l)]}{\pi(x+t-2 l)}\right)\left(\frac{\sin \left[\pi\left(x^{\prime}+t-2 l\right)\right]}{\pi\left(x^{\prime}+t-2 l\right)}\right)|x\rangle\left\langle x^{\prime}\right|,
$$

where $\left(\begin{array}{l}t \\ l\end{array}\right)=\frac{t !}{l !(t-l) !}$. From the properties of sine function, it is possible to argue that all the terms in (26) except $x=x^{\prime}=2 l-t$ term become zero. Thus, the explicit form of $\rho_{M 1 L}^{(x)}(t)$ can be written as

$$
\rho_{M 1 L}^{(x)}(t)=\sum_{x} \frac{t !}{2^{t}\left(\frac{t+x}{2}\right) !\left(\frac{t-x}{2}\right) !}|x\rangle\langle x|,
$$

where $x \in\{-t,-t+2, \ldots, t-2, t\}$. According to the expression given in (27) the offdiagonal terms of the position density matrix have vanished. In other words, the terms that contained information of coherence among the position states have become zero. This represents a quantum system subjected to perfect decoherence. In addition, note that $\rho_{M 1 L}^{(x)}(t)$ has no dependence on the initial coin state or the coin operator of the QW. Now let us determine the probability of finding the walker at point $y$ at time $t$ in the following way

$$
P_{M 1 L}(y, t)=\left\langle y\left|\rho_{M 1 L}^{(x)}(t)\right| y\right\rangle=\frac{t !}{2^{t}\left(\frac{t+y}{2}\right) !\left(\frac{t-y}{2}\right) !},
$$

where $y \in\{-t,-t+2, \ldots, t-2, t\}$. Note that the expression in (28) exactly equals to the probability mass function of an unbiased classical random walk [34]. Figure 1a shows the simulated and theoretical probability distributions of the one-dimensional Hadamard walk under the decoherence Model 1 with a localized initial state.

(a)

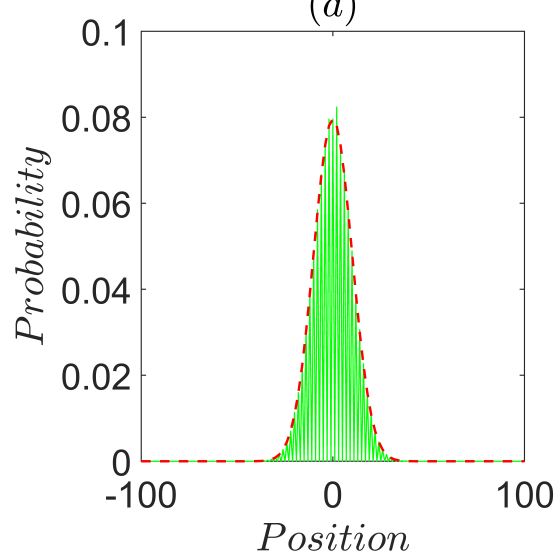

(b)

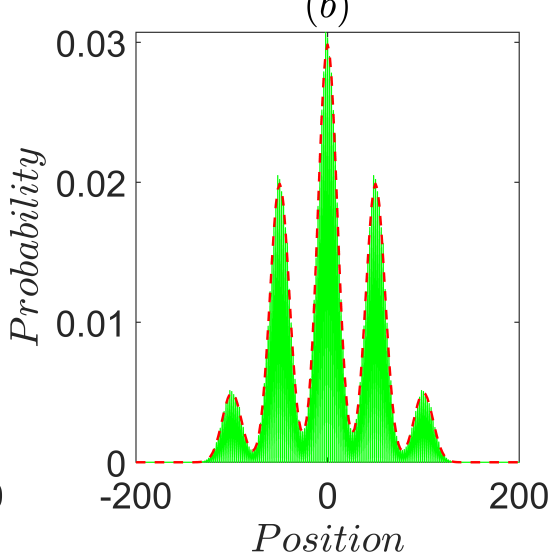

Figure 1. The simulated probability distributions of the one-dimensional Hadamard walk under the decoherence Model 1 for 100 time steps: (a) The walker is initiated from the localized state of $|0\rangle \otimes \frac{1}{\sqrt{2}}(|0\rangle+|1\rangle)$. The dashed line (red) shows the theoretical distribution. The standard deviation (10.03) is propositional to the square root of time steps and exhibits the signature of a classical random walk. (b) The walker is initiated from a delocalized state of $\sum_{x}|x\rangle \otimes\left(\alpha_{x}|0\rangle+\beta_{x}|1\rangle\right)$, where $x \in\{-100,-50,0,50,100\}$ and the corresponding $\left(\alpha_{x}, \beta_{x}\right)$ are $\left\{\left(\frac{1}{\sqrt{32}}, \frac{1}{\sqrt{32}}\right),\left(\frac{1}{\sqrt{8}}, \frac{1}{\sqrt{8}}\right),\left(\sqrt{\frac{3}{16}}, \sqrt{\frac{3}{16}}\right),\left(\frac{1}{\sqrt{8}}, \frac{1}{\sqrt{8}}\right),\left(\frac{1}{\sqrt{32}}, \frac{1}{\sqrt{32}}\right)\right\}$, respectively. The dashed line (red) shows the theoretical distribution. The standard deviation (50.99) seems to indicate a non-diffusive behavior. The time step and the initial position states are even and hence the walker resides only on even sites after the 100 time steps.

\subsection{Model 1 with Delocalized Initial State (M1D)}

Since $\mathcal{L}_{\hat{A}, k, k^{\prime}}$ is linear, operation of addition is preserved under $\mathcal{L}_{\hat{A}, k, k^{\prime}}$ and hence we can apply the superoperator on each outer product of the coin space separately. Then by 
tracing out the coin space from the density matrix of the coin-walker system given in (13) we can write the position density matrix under decoherence Model 1 with delocalized initial state $\rho_{M 1 D}^{(x)}(t)$ at time $t$ as follows

$$
\begin{aligned}
\rho_{M 1 D}^{(x)}(t)= & \operatorname{Tr}_{c}\left(\rho_{t}\right)=\sum_{x, x^{\prime}} \int_{-\pi}^{\pi} \frac{d k}{2 \pi} \int_{-\pi}^{\pi} \frac{d k^{\prime}}{2 \pi} e^{-i k x} e^{i k^{\prime} x^{\prime}}|k\rangle\left\langle k^{\prime}\right| \\
& \times\left[a_{x} a_{x^{\prime}}^{*} \operatorname{Tr}_{c}\left(\mathcal{L}_{\hat{A}, k, k^{\prime}}^{t}|0\rangle\langle 0|\right)+a_{x} b_{x^{\prime}}^{*} \operatorname{Tr}_{\mathcal{c}}\left(\mathcal{L}_{\hat{A}, k, k^{\prime}}^{t}|0\rangle\langle 1|\right)\right. \\
& \left.+b_{x} a_{x^{\prime}}^{*} \operatorname{Tr}_{\mathcal{c}}\left(\mathcal{L}_{\hat{A}, k, k^{\prime}}^{t}|1\rangle\langle 0|\right)+b_{x} b_{x^{\prime}}^{*} \operatorname{Tr}_{\mathcal{c}}\left(\mathcal{L}_{\hat{A}, k, k^{\prime}}^{t}|1\rangle\langle 1|\right)\right] .
\end{aligned}
$$

Note that from (21), for each initial coin state, the trace of the operator can be written as $\operatorname{Tr}\left(\mathcal{L}_{\hat{A}, k, k^{\prime}}^{t} O\right)=2 r_{0} \cos ^{t}\left(k-k^{\prime}\right)$ where $r_{0}$ is the coefficient corresponding to the coin state under the Pauli basis expansion. Using this result, it can be easily shown that $\operatorname{Tr}\left(\mathcal{L}_{\hat{A}, k, k^{\prime}}^{t}|0\rangle\langle 1|\right)=\operatorname{Tr}\left(\mathcal{L}_{\hat{A}, k, k^{\prime}}^{t}|1\rangle\langle 0|\right)=0$ and $\operatorname{Tr}\left(\mathcal{L}_{\hat{A}, k, k^{\prime}}^{t}|0\rangle\langle 0|\right)=\operatorname{Tr}\left(\mathcal{L}_{\hat{A}, k, k^{\prime}}^{t}|1\rangle\langle 1|\right)=$ $\cos ^{t}\left(k-k^{\prime}\right)$. Thus, the expression given in (29) can be rewritten as

$$
\rho_{M 1 D}^{(x)}(t)=2 r_{0} \sum_{x, x^{\prime}} \int_{-\pi}^{\pi} \frac{d k}{2 \pi} \int_{-\pi}^{\pi} \frac{d k^{\prime}}{2 \pi} e^{-i k x} e^{i k^{\prime} x^{\prime}}\left(a_{x} a_{x^{\prime}}^{*}+b_{x} b_{x^{\prime}}^{*}\right) \cos ^{t}\left(k-k^{\prime}\right)|k\rangle\left\langle k^{\prime}\right| .
$$

The probability of finding the walker at point $y$ at time $t$ is given by

$$
\begin{aligned}
P_{M 1 D}(y, t) & =\left\langle y\left|\rho_{M 1 D}^{(x)}(t)\right| y\right\rangle \\
& =\sum_{x, x^{\prime}} \int_{-\pi}^{\pi} \frac{d k}{2 \pi} \int_{-\pi}^{\pi} \frac{d k^{\prime}}{2 \pi} e^{-i k(x-y)} e^{i k^{\prime}\left(x^{\prime}-y\right)}\left(a_{x} a_{x^{\prime}}^{*}+b_{x} b_{x^{\prime}}^{*}\right) \cos ^{t}\left(k-k^{\prime}\right) .
\end{aligned}
$$

The expression given in (31) can be evaluated by expressing the cosine function in terms of complex exponential function and then by applying the binomial theorem. In doing so, we can obtain the probability of finding the walker at point $y$ at time $t$ as follows

$$
P_{M 1 D}(y, t)=\frac{1}{2^{t}} \sum_{x}\left(\left|a_{x}\right|^{2}+\left|b_{x}\right|^{2}\right) \frac{t !}{\left(\frac{t+x+y}{2}\right) !\left(\frac{t-x-y}{2}\right) !}
$$

where $x \in \mathbb{Z}$ and $y \in\{(-t+x),(-t+x+2), \ldots,(t+x-2),(t+x)\}$. The result obtained in (32) can be interpreted as a convex superposition of independent unbiased classical random walks. Figure $1 \mathrm{~b}$ shows the simulated and theoretical probability distributions of the one-dimensional Hadamard walk under the decoherence Model 1 with a delocalized initial state.

Let us explore in more detail the expression obtained in (32). Let $\left\{a_{x}\right\}_{x=-t}^{t}$ and $\left\{b_{x}\right\}_{x=-t}^{t}$ be sets of complex numbers such that $\sum_{x}\left|a_{x}\right|^{2}+\left|b_{x}\right|^{2}=1$. Write $S_{x}=\left|a_{x}\right|^{2}+$ $\left|b_{x}\right|^{2}$. Now let us denote the the probability of finding the quantum walker at the origin at time $t$ as $R_{t}$. Note that $R_{t}=P_{M 1 D}(0, t)$. Then the expression given in (32) can be viewed as a transformation or a data encoding scheme which maps the set $\left\{S_{x}\right\}$ to $\left\{R_{t}\right\}_{t \in \mathbb{N}}$ in the following way

$$
R_{t}=\frac{1}{2^{t}} \sum_{x} S_{x}\left(\begin{array}{c}
t \\
\frac{t+x}{2}
\end{array}\right)
$$

where $x \in\{-t,-t+2, \ldots, t-2, t\}$. Note that the term $2^{t} R_{t}$ gives the binomial transform of the set $\left\{S_{x}\right\}$ [44] and might be helpful, e.g., in identifying sequences which are invariant under its action [45].

\subsection{Model 2 with Localized Initial State (M2L)}

Following the analysis given in previous sections, one can determine the position density matrix under decoherence Model 2 with localized initial state by tracing out the 
coin space from the density matrix given in (10). The term $\operatorname{Tr}_{c}\left(\mathcal{L}_{\hat{E}, k, k^{\prime}}^{t}\left|\phi_{0}\right\rangle\left\langle\phi_{0}\right|\right)$ can be evaluated by summing the diagonal terms of the matrix given in (23). Hence, the position density matrix $\rho_{M 2 L}^{(x)}(t)$ in $x$-basis, can be expressed as

$$
\rho_{M 2 L}^{(x)}(t)=\frac{2 r_{0}}{(2 \pi)^{2}} \sum_{x, x^{\prime}} \int_{-\pi}^{\pi} d k \int_{-\pi}^{\pi} d k^{\prime} e^{i k x} e^{-i k^{\prime} x^{\prime}}\left(\Omega_{k, k^{\prime}}\right)^{t}|x\rangle\left\langle x^{\prime}\right|,
$$

where $\Omega_{k, k^{\prime}}=\cos \left(k-k^{\prime}\right)+i(1-2 p) \cos 2 \theta \sin \left(k-k^{\prime}\right)$. The definite integrals given in (34) can be evaluated by expressing the cosine and sine functions in terms of complex exponential function and then by applying the binomial theorem. Then, we obtain the following expression for $\rho_{M 2 L}^{(x)}(t)$ as

$$
\begin{aligned}
\rho_{M 2 L}^{(x)}(t) & =\sum_{x, x^{\prime}} \sum_{r=0}^{t}\left(\begin{array}{l}
t \\
r
\end{array}\right)\left(\frac{1+(1-2 p) \cos 2 \theta}{2}\right)^{r}\left(\frac{1-(1-2 p) \cos 2 \theta}{2}\right)^{t-r} \\
& \times\left(\frac{\sin [\pi(x+t-2 r)]}{\pi(x+t-2 r)}\right)\left(\frac{\sin \left[\pi\left(x^{\prime}+t-2 r\right)\right]}{\pi\left(x^{\prime}+t-2 r\right)}\right)|x\rangle\left\langle x^{\prime}\right|,
\end{aligned}
$$

where $\left(\begin{array}{l}t \\ r\end{array}\right)=\frac{t !}{r !(t-r) !}$. From the properties of sine function, it is possible to argue that all the terms in (35) except $x=x^{\prime}=2 r-t$ term become zero. Thus, the explicit form of $\rho_{M 2 L}^{(x)}(t)$ can be written as

$$
\begin{aligned}
\rho_{M 2 L}^{(x)}(t) & =\sum_{x} \frac{t !}{\left(\frac{t+x}{2}\right) !\left(\frac{t-x}{2}\right) !}\left(\frac{1+(1-2 p) \cos 2 \theta}{2}\right)^{\frac{t+x}{2}} \\
& \times\left(\frac{1-(1-2 p) \cos 2 \theta}{2}\right)^{\frac{t-x}{2}}|x\rangle\langle x|
\end{aligned}
$$

where $x \in\{-t,-t+2, \ldots, t-2, t\}$. The probability of finding the walker at point $y$ at time $t$ can be written as

$$
P_{M 2 L}(y, t)=\frac{t !}{2^{t}\left(\frac{t+y}{2}\right) !\left(\frac{t-y}{2}\right) !}(1+(1-2 p) \cos 2 \theta)^{\frac{t+y}{2}}(1-(1-2 p) \cos 2 \theta)^{\frac{t-y}{2}}
$$

where $y \in\{-t,-t+2, \ldots, t-2, t\}$. According to the set of Kraus operators given in (15), the parameter $p$ represents the impact of temperature on the process of losingor absorption of a photon. Hence, when $p=\frac{1}{2}$, the thermal influence on losing/absorbing a photon by the qubit system is equally probable. Note that, for the condition of $p=\frac{1}{2}$, one can obtain the relation of $P_{M 1 L}(y, t)=P_{M 2 L}(y, t)$ (see (28) and (37)). That is, the influence of the decoherence Model 1 and 2 on the QW is same. In addition, the expression (37) contains the coin parameter $\theta$ explicitly. When $p \neq \frac{1}{2}$, one can argue that, under the influence of the decoherence Model 2, probability distribution of the QW has a dependency on the coin parameter as well. This gives an ability to tune the probability distribution by changing the coin parameter. However, decoherence Model 1 does not show such a behaviour.

\subsection{Model 2 with Delocalized Initial State (M2D)}

By comparing the terms in expression (31) the probability of finding the walker at point $y$ at time $t$ can be written as

$$
\begin{aligned}
P_{M 2 D}(y, t) & =\left\langle y\left|\rho_{M 2 D}^{(x)}(t)\right| y\right\rangle \\
& =\sum_{x, x^{\prime}} \int_{-\pi}^{\pi} \frac{d k}{2 \pi} \int_{-\pi}^{\pi} \frac{d k^{\prime}}{2 \pi} e^{-i k(x-y)} e^{i k^{\prime}\left(x^{\prime}-y\right)}\left(a_{x} a_{x^{\prime}}^{*}+b_{x} b_{x^{\prime}}^{*}\right) \Omega_{k, k^{\prime}}^{t}
\end{aligned}
$$


where $\Omega_{k, k^{\prime}}=\cos \left(k-k^{\prime}\right)+i(1-2 p) \cos 2 \theta \sin \left(k-k^{\prime}\right)$. Following the same analysis given in the previous section, one can solve the integrals given in (38). Hence, an explicit expression for the probability $P_{M 2 D}(y, t)$ can be written as follows

$$
\begin{aligned}
P_{M 2 D}(y, t) & =\sum_{x}\left(\left|a_{x}\right|^{2}+|b|^{2}\right) \frac{t !}{2^{t}\left(\frac{t+x+y}{2}\right) !\left(\frac{t-x-y}{2}\right) !} \\
& \times(1+(1-2 p) \cos 2 \theta)^{\frac{t+x+y}{2}}(1-(1-2 p) \cos 2 \theta)^{\frac{t-x-y}{2}}
\end{aligned}
$$

where $y \in\{-t,-t+2, \ldots, t-2, t\}$. Note that when $p=\frac{1}{2}$ the result obtained in (39) becomes a convex superposition of independent unbiased classical random walks as given in (32).

\section{QW on a Cyclic Path}

A quantum walker that exhibits a one dimensional QW on a closed loop structure can be recognized as a cyclic QW. Consider a cyclic path consisting of $N$ discrete sites. The unitary operator $V$ that governs the evolution of the walker in the cyclic path is given by $V=\tilde{S}\left(\mathbb{I}_{x} \otimes C\right)$ where $C$ is the coin operator given in (2) and $\tilde{S}$ is the shift operator. For a cyclic QW, the shift operator is defined as follows

$$
\tilde{S}=\sum_{x=-\infty}^{\infty}|(x+1) \bmod N\rangle\langle x|\otimes| 0\rangle\langle 0|+|(x-1) \bmod N\rangle\langle x|\otimes| 1\rangle\langle 1| .
$$

The transformation from the $x$-basis to the $k$-basis is given by

$$
|k\rangle=\frac{1}{\sqrt{N}} \sum_{x} e^{i\left(\frac{2 \pi}{N}\right) k x}|x\rangle .
$$

The inverse transformation is given by

$$
|x\rangle=\frac{1}{\sqrt{N}} \sum_{k} e^{-i\left(\frac{2 \pi}{N}\right) k x}|k\rangle .
$$

Note that, the Born-von-Karman boundary conditions, otherwise known as the periodic boundary conditions, impose restrictions on the quasi-momenta, $k$ of the walker. Hence the $k$ values get the discrete form of $k_{m}=2 \pi m / N$ where $m$ ranges from 0 to $N-1$. As the number of nodes increases, the allowed $k$ values merge into a continuum [46]. Thus, in momentum space, $V$ is represented by the unitary operator $V_{k}$ given by

$$
V_{k}=\left(e^{-i\left(\frac{2 \pi}{N}\right) k} P_{R}+e^{i\left(\frac{2 \pi}{N}\right) k} P_{L}\right) C,
$$

where $P_{R}=|0\rangle\langle 0|$ and $P_{L}=|1\rangle\langle 1|$ are two projectors on coin Hilbert space and $C$ is the coin operator given in (2). In the context of cyclic QWs, the superoperator corresponding to flipping channels $\mathcal{L}_{\hat{A}, k, k^{\prime}}$ can be written as follows

$$
\mathcal{L}_{\hat{A}, k, k^{\prime}} O=\left(\begin{array}{cccc}
a_{11} & a_{12} & 0 & a_{14} \\
0 & a_{22} & a_{23} & a_{24} \\
0 & a_{32} & a_{33} & a_{34} \\
a_{41} & a_{42} & 0 & a_{44}
\end{array}\right)\left(\begin{array}{c}
r_{0} \\
r_{1} \\
r_{2} \\
r_{3}
\end{array}\right),
$$

$$
\begin{aligned}
& \text { where } a_{11}=(p+q+r+s) \cos \left[\frac{2 \pi\left(k-k^{\prime}\right)}{N}\right] \\
& a_{12}=-i(p+q-r-s) \sin \left[\frac{2 \pi\left(k-k^{\prime}\right)}{N}\right] \sin 2 \theta \\
& a_{14}=-i(p-q+r-s) \cos 2 \theta \sin \left[\frac{2 \pi\left(k-k^{\prime}\right)}{N}\right]
\end{aligned}
$$




$$
\begin{aligned}
& a_{22}=-(p+q-r-s) \cos \left[\frac{2 \pi\left(k+k^{\prime}\right)}{N}\right] \cos 2 \theta \\
& a_{23}=(p-q-r+s) \sin \left[\frac{2 \pi\left(k+k^{\prime}\right)}{N}\right] \\
& a_{24}=(p-q+r-s) \cos \left[\frac{2 \pi\left(k+k^{\prime}\right)}{N}\right] \sin 2 \theta \\
& a_{32}=-(p+q-r-s) \cos 2 \theta \sin \left[\frac{2 \pi\left(k+k^{\prime}\right)}{N}\right] \\
& a_{33}=-(p-q-r+s) \cos \left[\frac{2 \pi\left(k+k^{\prime}\right)}{N}\right] \\
& a_{34}=(p-q+r-s) \sin \left[\frac{2 \pi\left(k+k^{\prime}\right)}{N}\right] \sin 2 \theta \\
& a_{41}=-i(p+q+r+s) \sin \left[\frac{2 \pi\left(k-k^{\prime}\right)}{N}\right] \\
& a_{42}=(p+q-r-s) \cos \left[\frac{2 \pi\left(k-k^{\prime}\right)}{N}\right] \sin 2 \theta \\
& a_{44}=(p-q+r-s) \cos \left[\frac{2 \pi\left(k-k^{\prime}\right)}{N}\right] \cos 2 \theta .
\end{aligned}
$$

The superoperator corresponding to damping channels $\mathcal{L}_{\hat{E}, k, k^{\prime}}$ in cyclic QWs is given by

$$
\mathcal{L}_{\hat{E}, k, k^{\prime}} O=\left(\begin{array}{cccc}
e_{11} & e_{12} & 0 & e_{14} \\
e_{21} & e_{22} & e_{23} & e_{24} \\
e_{31} & e_{32} & e_{33} & e_{34} \\
e_{41} & e_{42} & 0 & e_{44}
\end{array}\right)\left(\begin{array}{l}
r_{0} \\
r_{1} \\
r_{2} \\
r_{3}
\end{array}\right)
$$

where $e_{11}=\cos \left[\frac{2 \pi\left(k-k^{\prime}\right)}{N}\right]+i \gamma(1-2 p) \cos 2 \theta \sin \left[\frac{2 \pi\left(k-k^{\prime}\right)}{N}\right]$

$e_{12}=-i \sqrt{1-\gamma} \sin 2 \theta \sin \left[\frac{2 \pi\left(k-k^{\prime}\right)}{N}\right]$

$e_{14}=i(\gamma-1) \cos 2 \theta \sin \left[\frac{2 \pi\left(k-k^{\prime}\right)}{N}\right]$

$e_{21}=\gamma(2 p-1) \sin 2 \theta \cos \left[\frac{2 \pi\left(k+k^{\prime}\right)}{N}\right]$

$e_{22}=-\sqrt{1-\gamma} \cos 2 \theta \cos \left[\frac{2 \pi\left(k+k^{\prime}\right)}{N}\right]$

$e_{23}=\sqrt{1-\gamma} \sin \left[\frac{2 \pi\left(k+k^{\prime}\right)}{N}\right]$

$e_{24}=-(\gamma-1) \sin 2 \theta \cos \left[\frac{2 \pi\left(k+k^{\prime}\right)}{N}\right]$

$e_{31}=\gamma(2 p-1) \sin 2 \theta \sin \left[\frac{2 \pi\left(k+k^{\prime}\right)}{N}\right]$

$e_{32}=-\sqrt{1-\gamma} \cos 2 \theta \sin \left[\frac{2 \pi\left(k+k^{\prime}\right)}{N}\right]$

$e_{33}=-\sqrt{1-\gamma} \cos \left[\frac{2 \pi\left(k+k^{\prime}\right)}{N}\right]$

$e_{34}=-(\gamma-1) \sin 2 \theta \sin \left[\frac{2 \pi\left(k+k^{\prime}\right)}{N}\right]$

$e_{41}=\gamma(2 p-1) \cos 2 \theta \cos \left[\frac{2 \pi\left(k-k^{\prime}\right)}{N}\right]-i \sin \left[\frac{2 \pi\left(k-k^{\prime}\right)}{N}\right]$

$e_{42}=\sqrt{1-\gamma} \sin 2 \theta \cos \left[\frac{2 \pi\left(k-k^{\prime}\right)}{N}\right]$

$e_{44}=-(\gamma-1) \cos 2 \theta \cos \left[\frac{2 \pi\left(k-k^{\prime}\right)}{N}\right]$.

Note that the set of expressions in (44) and (45) can be obtained by replacing the $k \rightarrow\left(\frac{2 \pi}{N}\right) k$ and $k^{\prime} \rightarrow\left(\frac{2 \pi}{N}\right) k^{\prime}$ in the superoperators (18) and (19). Hence, one can calculate the density operators of the cyclic QW under the influence of decohehrence Model 1 and 2 by replacing the variables $k$ and $k^{\prime}$ accordingly in the integrals (25), (30), (34) and (38). Note that, the integration sign must also be replaced with summation notation. The density matrix for the cyclic QWs that initiated from localized and delocalized states under decoherence Model 1 can be written as

$$
\begin{aligned}
& \rho_{M 1 L}^{(x)}(t)=\frac{2 r_{0}}{N} \sum_{x, x^{\prime}} \sum_{k, k^{\prime}} e^{i\left(\frac{2 \pi}{N}\right) k x} e^{-i\left(\frac{2 \pi}{N}\right) k^{\prime} x^{\prime}} \cos ^{t}\left(k-k^{\prime}\right)|x\rangle\left\langle x^{\prime}\right| \\
& \rho_{M 1 D}^{(x)}(t)=\frac{2 r_{0}}{N} \sum_{x, x^{\prime}} \sum_{k, k^{\prime}} e^{-i\left(\frac{2 \pi}{N}\right) k x} e^{i\left(\frac{2 \pi}{N}\right) k^{\prime} x^{\prime}}\left(a_{x} a_{x^{\prime}}^{*}+b_{x} b_{x^{\prime}}^{*}\right) \cos ^{t}\left(k-k^{\prime}\right)|k\rangle\left\langle k^{\prime}\right| .
\end{aligned}
$$


Likewise, the density matrix for the cyclic QWs under decoherence Model 2 can be written as

$$
\begin{aligned}
& \rho_{M 2 L}^{(x)}(t)=\frac{2 r_{0}}{N} \sum_{x, x^{\prime}} \sum_{k, k^{\prime}} e^{i\left(\frac{2 \pi}{N}\right) k x} e^{-i\left(\frac{2 \pi}{N}\right) k^{\prime} x^{\prime}}\left(\Theta_{k, k^{\prime}}\right)^{t}|x\rangle\left\langle x^{\prime}\right| \\
& \rho_{M 1 D}^{(x)}(t)=\frac{2 r_{0}}{N} \sum_{x, x^{\prime}} \sum_{k, k^{\prime}} e^{-i\left(\frac{2 \pi}{N}\right) k x} e^{i\left(\frac{2 \pi}{N}\right) k^{\prime} x^{\prime}}\left(a_{x} a_{x^{\prime}}^{*}+b_{x} b_{x^{\prime}}^{*}\right)\left(\Theta_{k, k^{\prime}}\right)^{t}|k\rangle\left\langle k^{\prime}\right|,
\end{aligned}
$$

where $\Theta_{k, k^{\prime}}=\cos \left[\frac{2 \pi\left(k-k^{\prime}\right)}{N}\right]+i \gamma(1-2 p) \cos 2 \theta \sin \left[\frac{2 \pi\left(k-k^{\prime}\right)}{N}\right]$. By solving (46) and (47) we can obtain the same expressions for the probability distributions of QW on a line given in (28), (32), (37) and (39) for the cyclic QW as well.

\section{Discussion and Conclusions}

We have studied the dynamics of linear and cyclic quantum walks under the influence of a noisy environment. Four qubit decoherence models are introduced to the coin degree of freedom of linear and cyclic QWs. These models include flipping channels (bit flip, phase flip and bit-phase flip), depolarizing channel, phase damping channel and generalized amplitude damping channel. When the walker initiates from a localized position state, the exact probability distribution of the classical unbiased random walk can be obtained for both linear and cyclic walks at all-time steps. In contrast, when the walker initiates from a delocalized position state the probability distribution of a mixture can be obtained for both types of walks at all-time steps. Accordingly, one can safely state that decoherence generated by the Model 1 and 2 has no dependency on the topology of the quantum walk. From the point of view of quantum algorithms, QWs that initiate from a delocalized state and propagate through a noisy environment may give rise to useful algorithmic applications. Such QWs can be used as a computational tool for encoding data and in our case can be employed for calculating the binomial transform of a given set of numbers. Some of the above theoretical predictions pertaining to cyclic quantum walks could be experimentally tested within the optical setup described in [46] when explicitly incorporating there the corresponding decoherence models. Since one of the original goals behind [46] was mimicking wavepacket dynamics in cyclic structures like the benzene molecule, our analysis here may help to analyze transport phenomena in such molecules, when immersed in a noisy environment.

Author Contributions: Conceptualization, M.N.J., A.N. and E.C.; methodology, M.N.J., A.N. and E.C.; formal analysis, M.N.J., A.N. and E.C.; investigation, M.N.J., A.N. and E.C.; writing-original draft preparation, M.N.J.; writing—review and editing, A.N. and E.C.; supervision, A.N. and E.C.; funding acquisition, E.C. All authors have read and agreed to the published version of the manuscript.

Funding: This research was funded by grant number FQXi-RFP-CPW-2006 from the Foundational Questions Institute and Fetzer Franklin Fund, a donor-advised fund of Silicon Valley Community Foundation, by the Israeli Innovation authority grant No. 73795 (Quantum Communication Consortium), by the Pazy foundation, by the Quantum Science and Technology Program of the Israeli Council of Higher Education and by the President Scholarship Program at Bar-Ilan University.

Institutional Review Board Statement: Not applicable.

Informed Consent Statement: Not applicable.

Data Availability Statement: All the supporting data and calculations are available upon request.

Conflicts of Interest: The authors declare no conflict of interest.

\section{References}

1. Kempe, J. Quantum random walks: An introductory overview. Contemp. Phys 2003, 44, 307-327. [CrossRef]

2. Shenvi, N.; Kempe, J.; Whaley, K.B. Quantum random-walk search algorithm. Phys. Rev. A 2003, 67, 052307. [CrossRef] 
3. Childs, A.M.; Cleve, R.; Deotto, E.; Farhi, E.; Gutmann, S.; Spielman, D.A. Exponential algorithmic speedup by a quantum walk. In Proceedings of the Thirty-Fifth Annual ACM Symposium on Theory of Computing, San Diego, CA, USA, 9-11 June 2003; Association for Computing Machinery: New York, NY, USA.

4. Berry, S.D.; Wang J.B. Quantum-walk-based search and centrality. Phys. Rev. A 2010, 82, 042333. [CrossRef]

5. Childs, A.M. Universal computation by quantum walk. Phys. Rev. Lett. 2009, 102, 180501. [CrossRef] [PubMed]

6. Childs, A.M.; Gosset, D.; Webb, Z. Universal computation by multiparticle quantum walk. Science 2013, 339, 791-794. [CrossRef]

7. Lloyd, S. Quantum coherence in biological systems. J. Phys. Conf. Ser. 2011, 302, 012037. [CrossRef]

8. Oliveira, A.C.; Portugal, R.; Donangelo, R. Decoherence in two-dimensional quantum walks. Phys. Rev. A 2006, 74, 012312. [CrossRef]

9. Hoyer, S.; Sarovar, M.; Whaley, K.B. Limits of quantum speedup in photosynthetic light harvesting. New J. Phys. 2010, $12,065041$. [CrossRef]

10. Wójcik, A.; Łuczak, T.; Kurzyński, P.; Grudka, A.; Gdala, T.; Bednarska-Bzdęga, M. Trapping a particle of a quantum walk on the line. Phys. Rev. A 2012, 85, 012329. [CrossRef]

11. Zhang, R.; Xue, P.; Twamley, J. One-dimensional quantum walks with single-point phase defects. Phys. Rev. A 2014, 89, 042317 . [CrossRef]

12. Schreiber, A.; Cassemiro, K.N.; Potoček, V.; Gábris, A.; Jex, I.; Silberhorn, C. Decoherence and disorder in quantum walks: From ballistic spread to localization. Phys. Rev. Lett. 2011, 106, 180403. [CrossRef]

13. Crespi, A. Localization properties of two-photon wave packets. Nat. Photonics 2013, 7, 322-328 [CrossRef]

14. Xue, P.; Qin, H.; Tang, B. Trapping photons on the line: Controllable dynamics of a quantum walk. Sci. Rep. 2014, 4, 4825. [CrossRef]

15. Kitagawa, T.; Rudner, M.S.; Berg, E.; Demler, E. Exploring topological phases with quantum walks. Phys. Rev. A 2010, 82, 033429. [CrossRef]

16. Kitagawa, T.; Broome, M.A.; Fedrizzi, A.; Rudner, M.S.; Berg, E.; Kassal, I.; White, A.G. Observation of topologically protected bound states in photonic quantum walks. Nat. Commun. 2012, 3, 1872. [CrossRef] [PubMed]

17. Mackay, T.D.; Bartlett, S.D.; Stephenson, L.T.; Sanders, B.C. Quantum walks in higher dimensions. J. Phys. A 2002, $35,2745$. [CrossRef]

18. Dur, W.; Raussendorf, R.; Kendon, V.M.; Briegel, H.J. Quantum walks in optical lattices. Phys. Rev. A 2002, 66, 052319. [CrossRef]

19. Kendon, V.; Tregenna, B. Decoherence in a quantum walk on a line. In Proceedings of the 6th International Conference on Quantum Communication, Measurement and Computing, Cambridge, MA, USA, 23-26 July 2002.

20. Kendon, V.; Tregenna, B. Decoherence can be useful in quantum walks. Phys. Rev. A 2003, 67, 042315. [CrossRef]

21. Brun, T.A.; Carteret, H.A.; Ambainis, A. Quantum-to-classical transition for random walks. Phys. Rev. Lett. 2003, 91, 130602. [CrossRef]

22. Brun, T.A.; Carteret, H.A.; Ambainis, A. Quantum walks driven by many coins. Phys. Rev. A 2003, 67, 052317. [CrossRef]

23. Brun, T.A.; Carteret, H.A.; Ambainis, A. Quantum random walks with decoherent coins. Phys. Rev. A 2003, 67, 032304. [CrossRef]

24. Shapira, D.; Biham, O.; Bracken, A.J.; Hackett, M. One-dimensional quantum walk with unitary noise. Phys. Rev. A 2003, 68, 062315. [CrossRef]

25. López, C.C.; Paz, J.P. Phase-space approach to the study of decoherence in quantum walks. Phys. Rev. A 2003, 68, 052305. [CrossRef]

26. Konno, N. A path integral approach for disordered quantum walks in one dimension. Fluct. Noise Lett. 2005, 5, 529-537. [CrossRef]

27. Kendon, V.; Sanders, B.C. Complementarity and quantum walks. Phys. Rev. A 2005, 71, 022307. [CrossRef]

28. Wang, J.; Manouchehri, K. Physical Implementation of Quantum Walks, 1st ed.; Springer: Berlin, Germany, 2013.

29. Romanelli, A.; Siri, R.; Abal, G.; Auyuanet, A.; Donangelo, R. Decoherence in the quantum walk on the line. Phys. A 2005, $347,137$. [CrossRef]

30. Kosik, J.; Buzek, V.; Hillery, M. Quantum walks with random phase shifts. Phys. Rev. A 2006, 74, 022310. [CrossRef]

31. Kendon, V. Decoherence in quantum walks-a review. Math. Struct. Comput. Sci. 2007, 17, 1169. [CrossRef]

32. Gönülol, M.; Aydiner, E.; Müstecaplığlu, Ö.E. Decoherence in two-dimensional quantum random walks with traps. Phys. Rev. A 2009, 80, 022336. [CrossRef]

33. Annabestani, M.; Akhtarshenas, S.J.; Abolhassani, M.R. Decoherence in a one-dimensional quantum walk. Phys. Rev. A 2010, 81, 032321. [CrossRef]

34. Montero, M. Classical-like behavior in quantum walks with inhomogeneous, time-dependent coin operators. Phys. Rev. A 2016, 93, 062316. [CrossRef]

35. Hizak, J.; Logozar, R. A derivation of the mean absolute distance in one-dimensional random walk. Teh. Glas. 2011, 5, 10-16.

36. Alberti, A.; Alt, W.; Werner, R.; Meschede, D. Decoherence models for discrete-time quantum walks and their application to neutral atom experiments. New J. Phys. 2014, 16, 123052. [CrossRef]

37. Ahlbrecht, A.; Cedzich, C.; Matjeschk, R.; Scholz, V.B.; Werner, A.H.; Werner, R.F. Asymptotic behavior of quantum walks with spatio-temporal coin fluctuations. Quantum Inf. Process 2012, 11, 1219-1249. [CrossRef]

38. Perets, H.; Lahini, Y.; Pozzi, F.; Sorel, M.; Morandotti, R.; Silberberg, Y. Realization of quantum walks with negligible decoherence in waveguide lattices. Phys. Rev. Lett. 2008, 100, 170506. [CrossRef] 
39. Broome, M.A.; Fedrizzi, A.; Lanyon, B.P.; Kassal, I.; Aspuru-Guzik, A.; White, A.G. Discrete single-photon quantum walks with tunable decoherence. Phys. Rev. Lett. 2010, 104, 153602. [CrossRef]

40. Xiao, L.; Cassemiro, K.N.; Potoček, V.; Gábris, A.; Jex, I.; Silberhorn, C. Higher winding number in a nonunitary photonic quantum walk. Phys. Rev. A 2018, 98, 063847. [CrossRef]

41. Nielsen, M.; Chuang, I. Quantum Computation and Quantum Information, 10th ed.; Cambridge University Press: Cambridge, UK, 2010.

42. Hou, L.-Z.; Fang, M.-F. Entanglement-assisted classical capacity of a generalized amplitude damping channel. Chin. Phys. Lett. 2007, 24, 2482.

43. Preskill, J. Lecture Notes for Physics 229: Quantum Information and Computation; California Institute of Technology: Pasadena, CA, USA, 1998.

44. Boyadzhiev, M.N. Notes on the Binomial Transform: Theory and Table with Appendix on Stirling Transform; World Scientific: Singapore, 2018.

45. Sun, Z.H. Invariant sequences under binomial transformation. Fibonacci Quart. 2001, 29, 324-333.

46. Nejadsattari, F.; Zhang, Y.; Bouchard, F.; Larocque, H.; Sit, A.; Cohen, E.; Fickler, R.; Karimi, E. Experimental realization of wave-packet dynamics in cyclic quantum walks. Optica 2019, 6, 174-180. [CrossRef] 